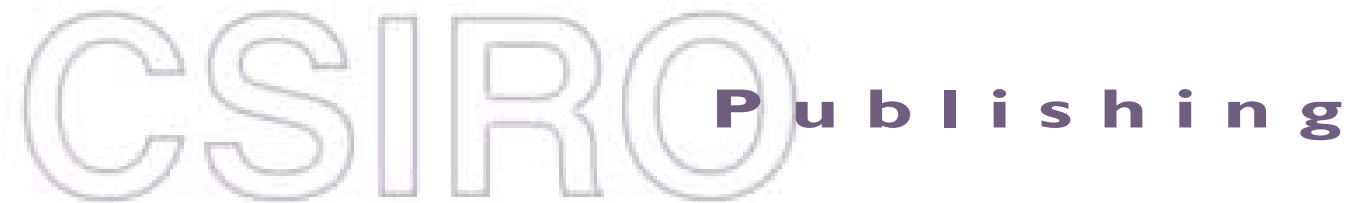

\section{Publications of the Astronomical Society of Australia}

Volume 19, 2002

(C) Astronomical Society of Australia 2002

An international journal of astronomy and astrophysics

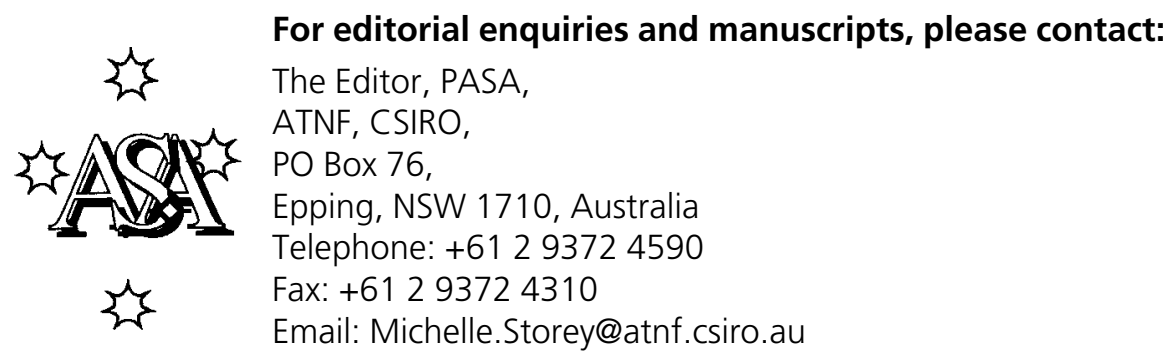

For general enquiries and subscriptions, please contact: CSIRO Publishing PO Box 1139 (150 Oxford St)

Collingwood, Vic. 3066, Australia

Telephone: +6139662 7666

Fax: +61 396627555

Email: publishing.pasa@csiro.au

C S I RO

PUBLISHING Published by CSIRO Publishing

for the Astronomical Society of Australia

www.publish.csiro.au/journals/pasa 


\title{
A Radio and Optical Study of the Active Young F Star HR 1817 (=HD 35850)
}

\author{
E. Budding ${ }^{1,2, *}$, B. D. Carter $^{3}$, M. W. Mengel $^{3}$, O. B. Slee ${ }^{4}$ and J.-F. Donati ${ }^{5}$ \\ ${ }^{1}$ Central Institute of Technology, PO Box 40740, Wellington, New Zealand \\ ${ }^{2}$ Carter Observatory, PO Box 2909, Wellington, New Zealand \\ ${ }^{3}$ Centre for Astronomy, Solar Radiation and Climate, Faculty of Sciences, \\ University of Southern Queensland, Toowoomba, Queensland 4350, Australia \\ carterb@usq.edu.au,mengelm@usq.edu.au \\ ${ }^{4}$ Australia Telescope National Facility, PO Box 76, Epping, NSW 1710, Australia \\ Bruce.Slee@atnf.csiro.au \\ ${ }^{5}$ Observatoire Midi-Pyrénées, 14 avenue Edouard Belin, F-31400 Toulouse, France \\ jean-francois.donati@ ast.obs-mip.fr \\ Received 2002 May 31, accepted 2002 September 6
}

\begin{abstract}
This paper presents the results of a multiwavelength observational study of the active young F-type star HR 1817. The star was monitored at 4.80 and $8.64 \mathrm{GHz}$ over $2 \times 12 \mathrm{~h}$ allocations with the Australia Telescope Compact Array on 8 and 9 December, 2000. The Anglo-Australian Telescope was used for simultaneous optical spectropolarimetry during a $2 \mathrm{~h}$ period on 9 December.

The low levels of observed radio emission have characteristics that are similar to those seen in other active stars, and a gyrosynchrotron mechanism is proposed to explain them; this is supported by the relatively low fractions of circular polarisation measured in HR 1817.

Comparison of the emissions from 4.80 and $8.64 \mathrm{GHz}$ shows a very strong cross-correlation peak, indicative of a common origin, although the shift of this peak indicates that $8.64 \mathrm{GHz}$ variations tend to precede those at $4.80 \mathrm{GHz}$ by, typically, $\sim 20 \mathrm{~min}$.

The optical spectropolarimetry reveals polarisation signals characteristic of surface magnetic fields, with profile changes indicating a complex dynamo-type magnetic topology is present on the star. This result makes HR 1817 the star with the earliest spectral type on which dynamo magnetic fields have been detected directly up to now.
\end{abstract}

Keywords: stars: activity — stars: coronae — stars: individual (HR 1817) — radio continuum: stars — techniques: miscellaneous — techniques: Zeeman Doppler imaging

\section{Introduction}

HR 1817 (HD 35850, HIP 25486) is a remarkably active, young, and relatively close single solar-type star, whose 6th magnitude brightness makes it convenient for studying the early history of Sun-like stars. This F7V object has rapid rotation, intense X-ray and extreme ultraviolet (EUV) emission, strong lithium abundance, a $v \sin i$ of $50 \mathrm{~km} \mathrm{~s}^{-1}$, a distance of $26.8 \mathrm{pc}$, and an age of $\approx 12 \mathrm{Myr}$ (Tagliaferri et al. 1997; Gagné et al. 1999; Mathioudakis \& Mullan 1999; Zuckerman et al. 2001). The star also has been identified recently as belonging to the young and nearby $\beta$ Pictoris moving group (Zuckerman et al. 2001). HR 1817 is slightly bigger and more massive than the Sun, with a quoted radius of $1.18 \mathrm{R}_{\odot}$ and mass of $1.15 \mathrm{M}_{\odot}$ (Gagné et al. 1999). The EUV observations suggest that HR 1817 is in a state of continuous low-amplitude flaring, providing a clear reason for interest in its radio behaviour (Gagné et al. 1999; Mathioudakis \& Mullan 1999). Activity in F stars is thought to relate to spectral peculiarities (e.g. Li anomalies: Rachford 1998; D’Antona et al. 2002; or line asymmetries: Dravins 1999), but there

\footnotetext{
* Present address: Canakkale Onsekiz Mart University (COMU), Turkey, ebudding@comu.edu.tr.
}

are unexplained variations from star to star, so it is useful also to acquire further spectroscopic data for stars of this class.

Microwave emission is a well-known feature of active stars, with radio emission detected for several dozen RS $\mathrm{CVn}$ binaries and several rapidly rotating and active single cool stars. This radio emission is thought to be produced by a gyrosynchrotron mechanism. The observed flux density correlates with rotation rate, which can be explained by the fact that increasingly rapid rotation enhances the dynamo generation of the magnetic fields ultimately responsible for the emission (Owen, Jones, \& Gibson 1976; Dulk 1985; Slee \& Stewart 1989).

Gyrosynchrotron radio emission from active stars is associated with moderate magnetic fields $(\sim 10-100$ gauss $)$ and high brightness temperatures $\left(\sim 10^{9} \mathrm{~K}\right.$, cf. e.g. Jones et al. 1996). Although it is usually impossible to give precise values for the magnetic field values just from radio observations, for HR 1817 a coronal magnetic field strength of $\mathrm{B} \leq 160$ gauss has been derived from analysis of EUV data by Mathioudakis \& Mullan (1999). In addition, surface fields can be detected and measured using an optical spectropolarimetric technique called Zeeman Doppler Imaging (ZDI) (Donati et al. 1997). 
Parallels might be drawn between HR 1817 and other active cool dwarfs. For example, multiwavelength studies of AB Dor revealed H $\alpha$ 'clouds' (Collier Cameron \& Robinson 1989) that relate to Mestel \& Spruit's (1987) coronal model. This model has been supported by a highdispersion spectroscopic study of the comparable star CS Cet (Watson 1999). Collier Cameron \& Robinson's (1989) picture of $\mathrm{AB}$ Dor would also be in keeping with previous microwave observations (Slee et al. 1986; Budding et al. 1992). Mestel \& Spruit's (1987) paper predicts the size of such coronal structures for a range of stellar parameters, and a natural question to ask is how well HR 1817 fits the model predictions. In addition, a study of CF Tuc (Budding et al. 1999) presented evidence of a strong correlation of radio emission across the range 2.8 to $8.4 \mathrm{GHz}$. That trend has since been confirmed in a number of other similar data sets (Budding et al. 2002), and a tendency to a phase delay in the cross-correlation curves has allowed inferences to be made about possible coronal mechanisms causing the delay. Another point of interest, then, is to consider how HR 1817's C-X band correlation properties compare to other active stars, and what this tells us about coronal emission mechanisms.

\section{Observations}

\subsection{Radio Observations}

The radio observations consisted of two $12 \mathrm{~h}$ allocations on the Australia Telescope Compact Array (ATCA) ${ }^{1}$ that started at $07 \mathrm{~h}$ UT on 8 December 2000, resuming on the next day at the same time. The discrete source PKS B1934-638 provided primary calibration of the flux levels at 4.80 and $8.64 \mathrm{GHz}$. The source PKS B0539-158 was conveniently placed for phase calibrations, and observed for 2-minute integrations, before 28-minute dwell times on HR 1817. Good phase stability was maintained throughout the observations. We observed only at $\mathrm{C} / \mathrm{X}$ bands because the emissions were not expected to exceed a few mJy, making it preferable to use frequencies at which the side lobes of possible confusing sources are minimal.

There are six $22 \mathrm{~m}$ dishes in the ATCA, accurately mounted on an east-west baseline. They can be arranged at appropriate separations to obtain specific angular resolutions at preassigned microwave observation frequencies (for further instrumental details cf. e.g. Manchester 1991). On this particular occasion, the telescopes were in the $1.5 \mathrm{E}$ configuration, which entailed appreciable shadowing of the two central closely spaced antennas for a few hours after the star became observable and before it set.

\subsection{Optical Observations}

Comparing radio and optical observations can provide a way of investigating the relationship between activity at different levels in a star's atmosphere. Optical spectropolarimetry enables study of the magnetic fields associated with surface activity, and the results compared

\footnotetext{
${ }^{1}$ The Australia Telescope National Facility is operated by CSIRO.
}

with coronal activity studied at microwave frequencies. Radio observations (BS \& EB) were thus made in conjunction with ZDI at the $3.9 \mathrm{~m}$ Anglo-Australian Telescope (AAT) (BC, JFD \& MM).

At the AAT, the Semel spectropolarimeter visitor instrument was used, with an instrumental setup similar to that described by Donati et al. (1997). Optics placed at the Cassegrain focus of the telescope produced circularly polarised light, guided to the University College London échelle spectrograph via a pair of optical fibres. An image slicer was used in the slit room of the spectrograph, to maximise light throughput from the fibres into the spectrograph optics. The data were recorded on the MIT/LL CCD detector.

The observations were made on 9 December 2000, almost continuously from approximately $10 \mathrm{~h}$ to $12 \mathrm{~h} \mathrm{UT}$. The observations consisted of a series of $300 \mathrm{~s}$ exposures, with switching of the beam polarisation employed to remove instrumental polarisation effects when the data are processed.

\section{Data Analysis and Results}

\subsection{Radio Data}

The ATCA data were conveniently handled on-site using the MIRIAD data reduction package (Sault \& Killeen 1996). The full available instrumental bandwidth for these microwave ranges is $128 \mathrm{MHz}$. Our processing procedure made use of the 'birdie' option in the MIRIAD routine ATLOD, which permits $13 \times 8 \mathrm{MHz}$ independent, interference-free, non-overlapping channels to be used for either band, summing to $104 \mathrm{MHz}$ of total bandwidth. The flux levels used to construct the light curves, discussed in what follows, were obtained from sky maps produced from the recorded visibility data via the MIRIAD data reduction suite.

A map of the field (Figure 1) shows three clear radio sources including HR 1817 . The measurements are summarised in Table 1. Each source was fitted with a 2-dimensional elliptical Gaussian superposed on a linear baseline, from which positions, flux densities (background subtracted), and angular sizes at the mean frequency of $4.80 \mathrm{GHz}$ were determined.

All three sources were unresolved. The position errors in the fitting are $0.2 \mathrm{~s}$ in RA and 0.'5 in dec, while flux densities have a formal error of $\sim 0.1 \mathrm{mJy}$. Our measured position for HR 1817 may be compared with that of the Hipparcos catalogue, i.e. (J2000) RA $5 \mathrm{~h} 27 \mathrm{~m} 4.76 \mathrm{~s}$; dec $-11^{\circ} 54^{\prime} 3.5^{\prime \prime}$. A digitised image from the Science and Engineering Research Council sky survey was searched for optical identifications for sources 2 and 3; source 2 is coincident with a galaxy having $\mathrm{m}_{R}=19.5$, but source 3 is unidentified.

\subsubsection{Flux Density versus Time}

The low-level flux densities of HR 1817 were finalised after lengthy review. First, we summed the integrations to about 2 hours to obtain more reliable statistics and 


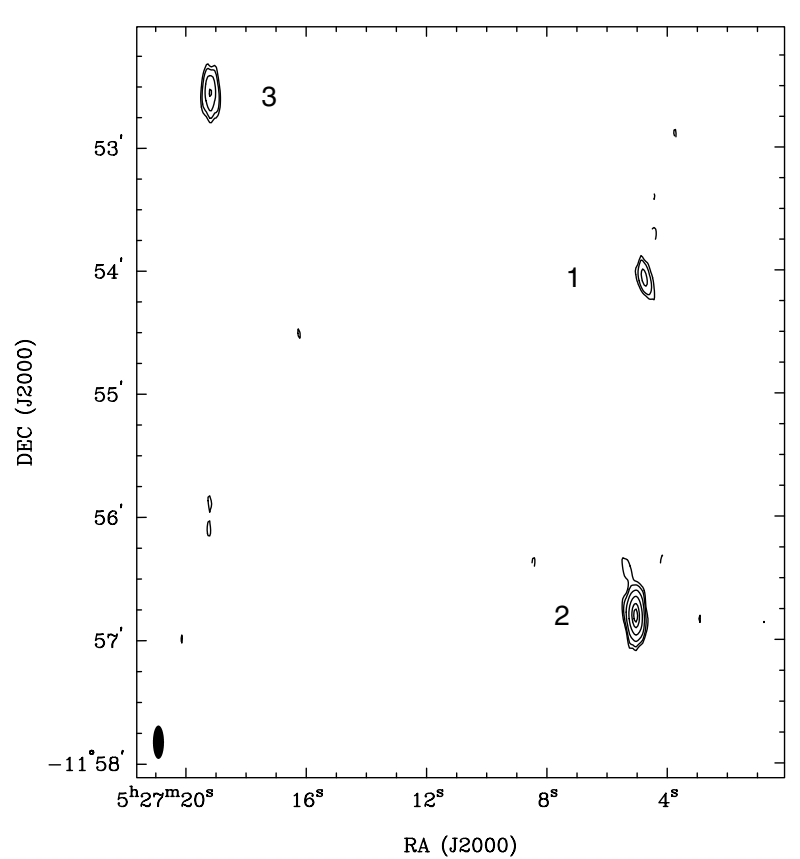

Figure 1 A cleaned $4.80 \mathrm{GHz}$ MIRIAD field map of the region near HR 1817 (source No.1) from the data of 8 December 2000, showing the two nearest field sources $(2 \& 3)$. The contour levels are $-6,6,10,20,40,70, \& 90$ per cent of the peak of $2.00 \mathrm{mJy}$ for source No. 2. The restoring beam in the lower left corner is $16.3 \times$ 5.4 arcsec to FWHM in PA $=0.1$ degrees. The PA of the major axis of HR 1817 is associated with an increase in flux density visible in the post-culmination data for 8 December in Figure 2.

Table 1. Radio sources in the HR 1817 field: Gaussian fit positions and flux densities

\begin{tabular}{|c|c|c|c|c|c|c|c|}
\hline \multirow[t]{3}{*}{ Source No. } & \multicolumn{6}{|c|}{ Position J2000 } & \multirow{3}{*}{$\begin{array}{c}\text { Flux density } \\
\text { (mJy) } \\
4.80 \mathrm{GHz}\end{array}$} \\
\hline & \multicolumn{3}{|c|}{ RA } & \multicolumn{3}{|c|}{ dec } & \\
\hline & $\mathrm{h}$ & $\mathrm{m}$ & $\mathrm{s}$ & $\circ$ & ' & " & \\
\hline 1 & 5 & 27 & 04.76 & -11 & 54 & 3.9 & 0.42 \\
\hline 2 & 5 & 27 & 05.04 & -11 & 56 & 48.4 & 2.13 \\
\hline 3 & 5 & 27 & 19.21 & -11 & 52 & 33.6 & 0.84 \\
\hline
\end{tabular}

Source No. 1 is HR 1817, 8 December 2000. Position errors are $\sim 0.5$ arcsec.

a preliminary overall impression. We utilised both 'peak' and 'profile' fittings to point source images, using the MIRIAD MAXFIT procedure. We then examined the individual 28 -min integrations, though these were not always well-defined, particularly in the first few hours of either day. (Data were somewhat compromised at low altitudes, due to the shadowing.)

The data on HR 1817 consisted of 21 individual integrations on each of the two nights. The flux densities shown in Figure 2 were derived from the MIRIAD task UVFIT, after subtracting the contributions of the field sources to the UV visibilities. In the subtraction process, the field was modelled from the clean components, with the area contributing to the clean components of HR 1817 being
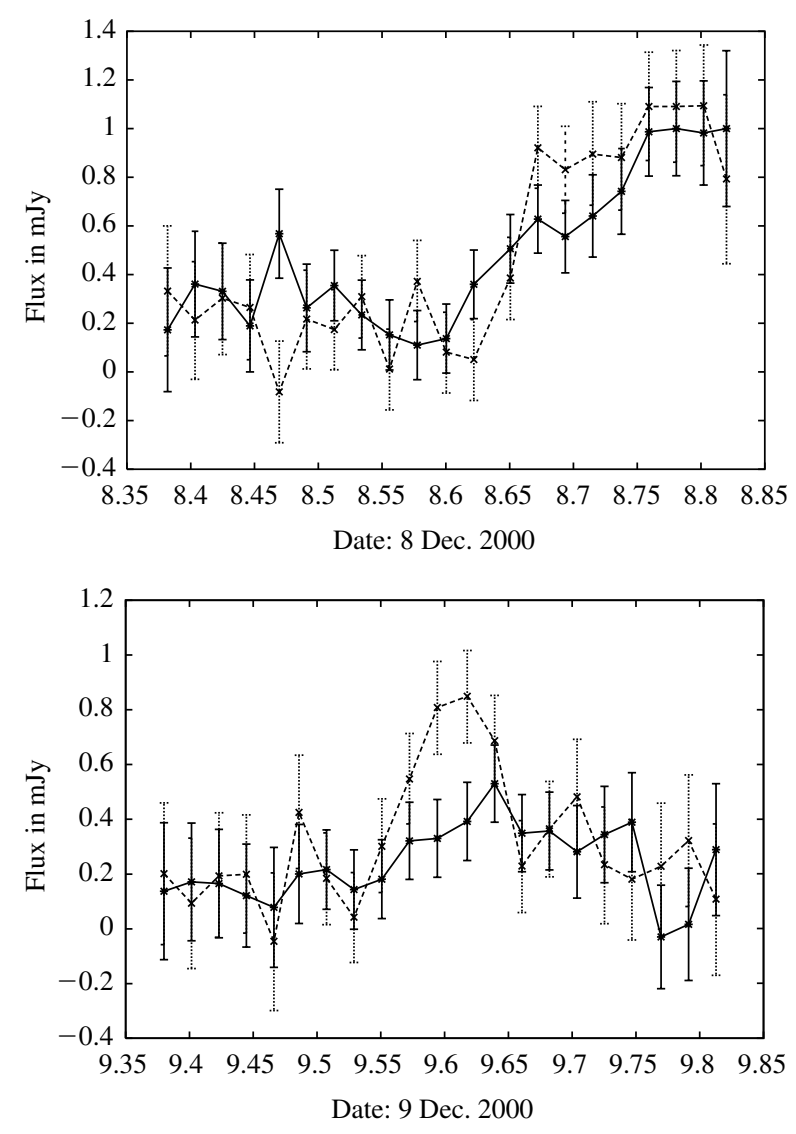

Figure 2 The 4.80 (continuous line) and 8.64 (dashed line) $\mathrm{GHz}$ flux densities are plotted, with error bars, against decimal UT date, corresponding to the individual integrations on both days.

masked. Such a process was found effective for reliable measurement of these very weak flux densities in the presence of side lobes from field sources.

The combined data curves (Figure 2) show that the emission level was generally higher on 8 December than on 9 December; the quiescent levels were $\sim 0.3$ and $\sim 0.2 \mathrm{mJy}$ on the two nights, respectively. The intensity increases to $\sim 1.1 \mathrm{mJy}$ towards the end of 8 December, with a fairly flat radio spectrum, and appears to maximise at $\sim 19.0$ UT. The flux density on 9 December rises significantly (especially at $8.64 \mathrm{GHz}$ ) to $0.5-0.8 \mathrm{mJy}$, some $20 \mathrm{~h}$ later, to a broad maximum at $\sim 15.0 \mathrm{~h}$.

\subsubsection{Correlation Effects}

Observations of the RS CVn binary CF Tuc in 1996 (Budding et al. 1999) showing 'quiescent' microwave fluctuations of $1-2 \mathrm{mJy}$, at 4.8 and $8.64 \mathrm{GHz}$, led to studies of the auto- and cross-correlation properties of the two data sets. The results encouraged further investigation (Budding et al. 2002) of more extensive paired sets of microwave data on selected chromospherically active stars. Comparisons were also made with empirical test data.

The auto-correlation contains information about the predominating timescales of changes in flux density. It 


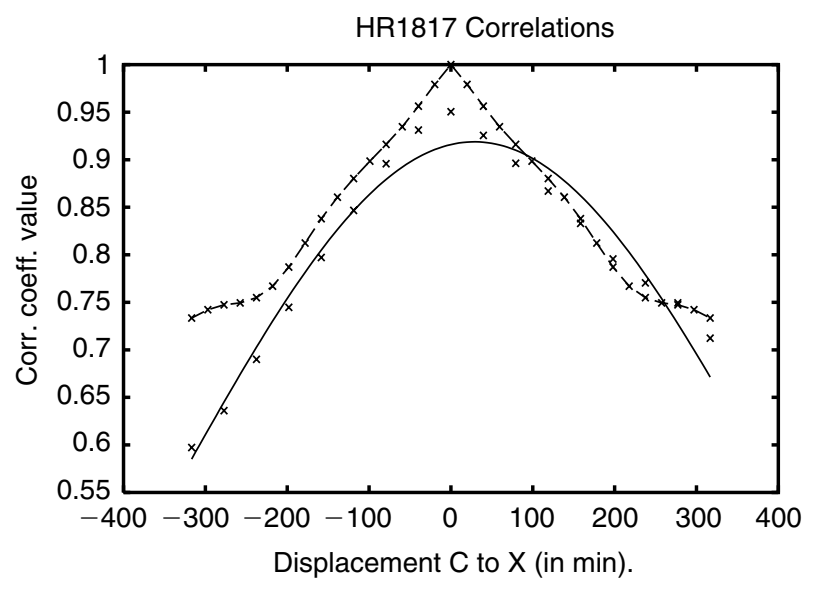

Figure 3 Correlation functions (auto - dashed line, cross continuous line) are here plotted for the data of 8 December 2000 , using the method discussed in Budding et al. (2002) (see Section 3.1.2).

shows, apart from a central narrow peak, generally broad forms for the 4.80 and $8.64 \mathrm{GHz}$ data sets, reflecting a rather long timescale of signal undulations, compared with available time resolutions. The cross-correlation measures the temporal relationship between flux density changes in the $\mathrm{C}$ and $\mathrm{X}$ bands.

Correlations (both auto and cross) are evaluated by shifting one (evenly spaced) data set with respect to the other, one interpolation interval at a time, and computing the relevant correlation product-sum at each shift. These values have then been plotted and fitted with Gaussians. This yields the axes of symmetry, widths, and formal errors. Different procedures were sometimes followed, but the results were generally found to be in close agreement. There is often a noticeable asymmetry to the cross-correlation, frequently with the curve's peak shifted to lags that imply intensity variations at $4.80 \mathrm{GHz}$ occurring some minutes after those at $8.64 \mathrm{GHz}$.

Tests with artificial data, arranged over appropriate interval and bin sizes, confirmed that shifts of the crosscorrelation maximum can be effective in measuring a real time delay between physically correlated data sets, even for relatively low $S / N$ data. These tests also showed that the value of the cross-correlation peak obtained for the real data sets is consistent with the pairs of flux density values being of essentially the same origin, but perturbed by random noise to an extent predictable from the known $S / N$ ratio.

We have carried out these procedures again for the $\mathrm{C}$ and $\mathrm{X}$ data sets of the present study, and the results are shown in Figure 3 (tabular data available on request). The auto-correlation (dashed line) suggests the contributory role of undulations on the order of $\sim 0.15 \mathrm{~d}$ or less. The cross-correlation (continuous line) indicates a high degree of similarity of the $\mathrm{X}$ to the $\mathrm{C}$ band flux densities (cf. Figure 2) and, given the inherent errors of observation, that they very probably originate in the same excitation mechanism.
The cross-correlation is somewhat asymmetric, but here has been fitted with a Gaussian. The shift of the axis of this Gaussian to positive lags of the $\mathrm{C}$ behind that of $\mathrm{X}$ band data is characteristic for this kind of source (cf. Budding et al. 2002). It is taken to indicate that the excitation tends to propagate outward in waves through the emitting corona. On this basis, variations in the layers contributing most to the $\mathrm{X}$ band emission occur typically $\sim 20$ min before those associated with the $\mathrm{C}$ band.

\subsubsection{Radio Polarisation}

The signal/noise ratios achieved by integrating the nightly data sets were high enough to set upper limits $3 \sigma$ on the fractional circular polarisation. For 8 December, the circular fractions were less than 0.25 at both 4.80 and $8.64 \mathrm{GHz}$. On 9 December, these fractions were less than 0.44 at $4.80 \mathrm{GHz}$ and less than 0.32 at $8.64 \mathrm{GHz}$. These moderate upper limits suggest that the emission is probably not due to a coherent mechanism, for which polarised fractions approaching unity are commonly observed.

\subsection{Optical Data}

Analysis of the optical data generated a polarisation spectrum by intercomparison of individual spectra taken in circularly polarised light. Almost three thousand photospheric lines in each spectrum were summed using a technique called least squares deconvolution, to improve signal to noise, and so provide Stokes $\mathrm{V}$ polarisation measurements accurate to about 1 part in $10^{5}$. This type of analysis has been described in more detail by Donati et al. (1997).

The optical measurements of HR 1817, taken simultaneously with the radio monitoring on 9 December 2000, confirm the presence of the star's surface magnetic field (Figure 4$)$, with a definite $(>10 \sigma)$ detection provided for every observation made. The data span a 2-hour period from around $10 \mathrm{~h} \mathrm{UT}$ to $12 \mathrm{~h}$ UT. Four sets of measurements were made, starting at 9:56, 10:20, 11:11, and 11:35 UT. Each measurement consisted of a sequence of 4 individual 300 s exposures, combined to produce a composite line polarisation spectrum, as well as a composite line intensity spectrum.

In each of these four measurements, the signature of the field is revealed as an asymmetrical distortion in the polarisation spectrum, at radial velocities corresponding to the star's surface. A comparison of the four polarisation profiles shows significant variation throughout the 2-hour period during which the optical data were collected. This variation in the polarisation signature corresponds to observation of the star at different phases of its rapid rotation.

The corresponding Stokes I composite line intensity profile for each observation shows a flat base towards the centre of the profile. This profile indicates a small but significant variability corresponding to shifts in the observed phase of stellar rotation. 
LS deconvolved Zeeman signature (2873 lines used)

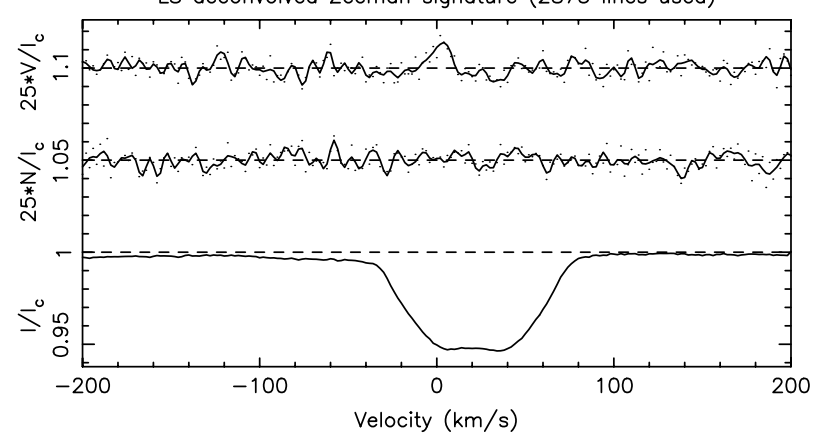

LS deconvolved Zeeman signature (2875 lines used)

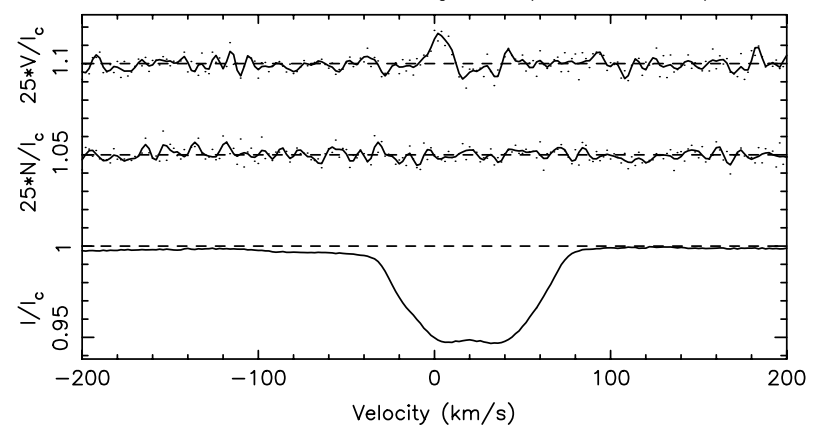

LS deconvolved Zeeman signature (2875 lines used)

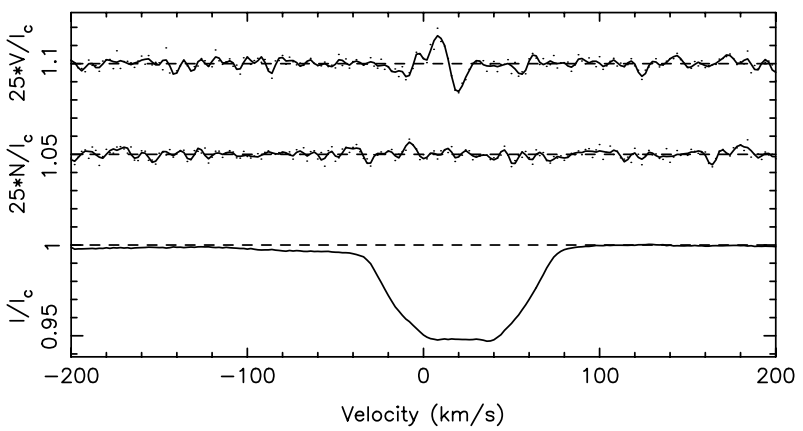

LS deconvolved Zeeman signature (2875 lines used)

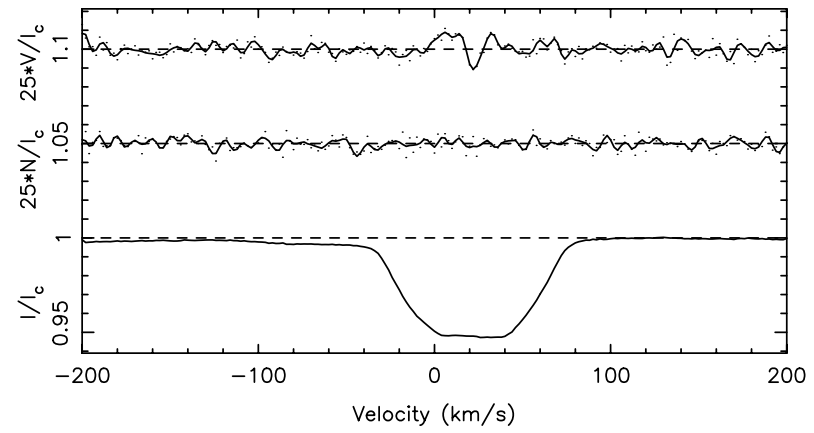

Figure 4 The optical polarisation and intensity profiles observed for HR 1817 on 9 December 2000 . The 4 frames cover a 2 hour period from around $10 \mathrm{~h} \mathrm{UT}$ to $12 \mathrm{~h} \mathrm{UT}$, with each frame corresponding to four $300 \mathrm{~s}$ exposures. From top to bottom, the UT start times for each frame are 9:56,10:20,11:11, and 11:35 UT. In each frame the uppermost plot is the Stokes $\mathrm{V}$ polarisation for the composite line profile. This displays the characteristic signature of a surface magnetic field. The middle plot shows the small noise level for the observation. The lowermost plot in each frame is the composite Stokes I line intensity profile. Its flat-bottomed shape suggests (though does not prove) that a dark spot is present.

\section{Discussion}

\subsection{Radio Emission Mechanism}

The radio observations presented here indicate emission that varies between 0.2 and $1.1 \mathrm{mJy}$ in a regular manner over a timescale of $\sim 20 \mathrm{~h}$. This is in keeping with broadly phase dependent emission from a localised active coronal source of diameter $\sim 0.1$ of the stellar circumference, and a stellar rotation period close to one day.

Although imprecise, the rotation period suggested by the radio results can be compared to previous work provided by satellite monitoring of the star in the extreme ultraviolet. Our finding is more consistent with the figure of almost exactly one day apparently favoured by Mathioudakis \& Mullan (1999), than the $1.40 \pm 0.20$ day period quoted by Gagné et al. (1999). For active stars, optical photometry of starspot modulation normally resolves the issue. However, HR 1817 was one of the few stars in a major photometric monitoring survey where no rotation period was obtained (Cutispoto, Messina, \& Rodono 2001). Photometry of the star obtained during the season concurrent with the radio observations (A. Erdem 2001, private communication) indicated slow drifts of $\sim 0.04$ mag in $V$ over timescales of order $\sim 3-4 \mathrm{~h}$. Photometry from the Hipparcos satellite is consistent with starspot maculation effects, but these data are insufficient to derive a meaningful period. A definitive value for HR 1817's rotation period remains an issue to be resolved.

The radio correlation results are generally consistent with a release of energy propagating by magnetohydrodynamic waves through an active corona, although the lags and leads between one data set and the other vary appreciably in magnitude and sometimes direction. The $\mathrm{X}$ to $\mathrm{C}$ band time-shifts for AB Dor are also noticeably shorter than those obtained for classical RS CVn binaries. This is in general keeping with larger scale coronal structures that one might expect for subgiants.

The results may be considered in terms of gyrosynchrotron radiation from a population of mildly relativistic electrons - a common way to interpret active star radio emission. Dulk's (1985) treatment gives very useful sets of summarising formulae for such interpretation. We first write, for the microwave flux $F_{v}$,

$$
F_{v}=2 \pi \frac{k v^{2}}{c^{2}} T_{b} A,
$$

where $T_{b}$ is the brightness temperature of a source of area $A$ and other symbols have their usual meanings. We can infer an approximate value for $T_{b}$, by assuming a coronal source size $\sim 0.1$ of the star's circumference or $A \sim 3 \times 10^{16} \mathrm{~m}^{2}$ (see above). We obtain the total flux at the source to be $\sim 8.6 \times 10^{7} \mathrm{~W} \mathrm{~Hz}^{-1}$ per mJy of received flux density. This then yields a brightness temperature of more than $\sim 10^{10} \mathrm{~K}$ for the source. This is high for normal gyrosynchrotron emission, but the calculation is quite dependent on the assumed value of $A$, which may well be larger for the coronal region involved than the assumed 
surface maculation size, bringing the temperature down to a more typical gyrosynchrotron range (say, $\sim 10^{9} \mathrm{~K}$ ).

Approximate formulae supplied by Dulk (1985) permit insights into the emission regime. Thus, Dulk's opacity equation (36), summed along a length $l$ down to a $\tau=1$ surface, can be put in logarithmic form. Taking a representative viewing angle as $\pi / 4$ and a power-law index $\delta=3$ for the electron energy spectrum, we then find

$$
20.65=\log l-4.24 \log v_{\mathrm{G}}+\log N_{e}+3.24 \log B,
$$

where $v_{\mathrm{G}}$ is the frequency in $\mathrm{GHz}, N_{e}$ is the radiating electron density, and $B$ is the magnetic induction (cgs units).

Dulk's (1985) equation (37) for the effective temperature can be used in equation (1) above, since we are supposing now an optically thick source, so that, for $1 \mathrm{mJy}$ of received flux, we have the relation

$$
22.08=2.76 \log v_{\mathrm{G}}-0.76 \log B+2 \log L,
$$

where $L$ is a representative radius of the emitting area.

A limiting case is obtained by setting $l=L$ in the foregoing, corresponding to the minimum size of emission region which can just become optically thick for a particular density. We may also add the constraint

$$
\log N_{e}=\log B+\text { constant, }
$$

assuming that the magnetic field is convected with the coronal plasma. The constant is $\approx 5.5$ for the solar corona. This approximation results in the values: $N_{e} \sim 1.9 \times 10^{7}, B \sim 62 \mathrm{G}, L \sim 6.0 \times 10^{10} \mathrm{~cm}$ at $\mathrm{C}$ band, and $N_{e} \sim 3.4 \times 10^{7}, B \sim 107 \mathrm{G}, L \sim 3.3 \times 10^{10} \mathrm{~cm}$ at $\mathrm{X}$ band (cf. Budding et al. 1999). Though these length-scales are large, they would still fit within the co-rotating 'dead zone' region of the corona of HR 1817, according to the modelling of Mestel \& Spruit (1987, Table 1: $\alpha / \alpha \odot \sim 30$, for which $\bar{r} / R \sim 4-6$ for a wide range of feasible surface field strengths).

Our limiting case scenario may be compared with that of Mathioudakis \& Mullan (1999), who consider a more compact, near-surface structure corresponding to $N_{e} \lesssim 5 \times 10^{11}, B \leq 160 \mathrm{G}, L \sim 4.0 \times 10^{9} \mathrm{~cm}$, based on ultraviolet emission from the loop foot regions.

We can speculate that the observed enhancements are powered, primarily, by strong flare-related excitations, as the existence of this behaviour will lead to the gyrosynchrotron emission associated with RS CVn stars. Continued detailed studies, with improved data quality and quantity, should help clarify the emission processes in the atmosphere of HR 1817.

\subsection{Surface Magnetic Field}

The optical data presented here confirm the presence of surface magnetic fields on HR 1817. Based on previous experience with ZDI of other stars, the observed temporal variability of the polarisation profile can be explained by the presence of complex surface fields. This is because the appearance of the profile is sensitive to the precise phase of rotation at which the star is observed. It seems that HR 1817, like the Sun, has a complex surface magnetic field topology. This result also makes HR 1817 the star with the earliest spectral type on which dynamo magnetic fields have been detected directly up to now (cf. Donati et al. 1997). HR 1817 thus shows that we can empirically study the magnetism of stars whose dynamos are of considerable interest, because of their relatively shallow convective zones (Gagné et al. 1999).

The flat base of the optical line intensity composite profile for each observation suggests (though does not prove) that a polar spot is present. If correct, this contrasts with the Sun's exclusively low and mid-latitude spot features, but is consistent with the polar spots observed for rapidly rotating active single $\mathrm{K}$ dwarfs such as $\mathrm{AB}$ Dor and LQ Hya.

The modest data set obtained from the optical spectropolarimetry is too limited in time coverage to generate a magnetic map, and hence estimate surface field strengths. The uncertain, but probable, one-day rotation period also raises the issue of how to obtain full phase coverage for the star. Nevertheless, given that definite magnetic detections can be made, it demonstrates the feasibility of using more extensive optical spectropolarimetry to map the star and quantitatively measure the star's surface fields.

\section{Conclusion}

We conclude that HR 1817 is a single young solar-type star whose remarkable magnetic activity is evident at radio wavelengths, and directly observable in the optical. Furthermore, given the similarities of HR 1817's radio emission to that of RS CVn binaries, it is reasonable to propose that a similar physical process is responsible. Thus, we speculate that the radio emission of HR 1817, like that of RS CVn systems, is powered primarily by strong flare-related excitations leading to gyrosynchrotron emission.

HR 1817 is clearly an object deserving of further study. Continued detailed radio studies, with improved data quality and quantity, should help confirm the presence of a gyrosynchrotron emission mechanism in the corona. In addition, further optical spectropolarimetry, involving different telescopes spread out in longitude, could help considerably with the study of stars like HR 1817 . With such data, one could build up a complete magnetic map of the star's photosphere, which in turn could be extrapolated to derive a simplified, 3-dimensional picture of the coronal magnetic field. Such an approach should assist efforts to relate more quantitatively data from optical and radio domains, and provide new information on where and how radio emission is produced in the magnetospheres of solar-type stars.

\section{Acknowledgements}

EB acknowledges the support of the CIT's Research and Scholarship Committee and the Bilateral Research 
Association Programme (BRAP) of the Royal Society of New Zealand in supporting his observational visit to the Australia Telescope National Facility. BC, JF, and MM would like to extend sincere thanks to the Director and Staff of the Anglo-Australian Observatory, and to Meir Semel of the Observatoire Paris-Meudon for use of the ZDI SemelPol polarimeter. The AAT observations of BC and $\mathrm{MM}$ were supported by a team project grant provided by the University of Southern Queensland. This paper made use of data from the Hipparcos satellite, the Science and Engineering Research Council, SIMBAD, and the NASA Astrophysics Data System.

\section{References}

Budding, E., Burgess, A., Chan, S., \& Slee, O. B. 1992, in Surface Inhomogeneities on Late Type Stars, Lecture Notes in Physics 397, ed. P. B. Byrne \& D. J. Mullan (Heidelberg: Springer-Verlag), 253

Budding, E., Jones, K. L., Slee, O. B., \& Watson, L. 1999, MNRAS, 305, 966

Budding, E., Lim, J., Slee, O. B., \& White, S. M. 2002, NewA, 7, 35

Collier Cameron, A., \& Robinson, R. D. 1989, MNRAS, 238, 657

Cutispoto, G., Messina, S., \& Rodono, M. 2001, A\&A, 367, 910
D’Antona, F., Montalban, J., Kupka, F., \& Heiter, U. 2002, ApJ, 564, L93

Donati, J.-F., Semel, M., Carter, B. D., Rees, D. E., Collier Cameron, A. 1997, MNRAS, 291, 658

Dravins, D. 1999, in ASP Conf. Series 185, IAU Colloquium 170, ed. J. B. Hearnshaw \& C. D. Scarfe (San Francisco: ASP), 268 Dulk, G. A. 1985, ARA\&A, 23, 169

Gagné, M., Valenti, J. A., Linksy, J. L., Tagliaferri, G., Covino, S., \& Güdel, M. 1999, ApJ, 515, 423

Jones, K. L., Brown, A., Stewart, R. T., Slee, O. B. 1996, MNRAS, 283, 1331

Mathioudakis, M., \& Mullan, D. J. 1999, A\&A, 342, 524

Manchester, R. 1991, AdSpR, 11, 403

Mestel, L., \& Spruit, H. C. 1987, MNRAS, 226, 57

Owen, F. N., Jones, T. W., \& Gibson, D. M. 1976, ApJ, 210, L27

Rachford, B. L. 1998, PhD Thesis, University of Wyoming

Sault, R., \& Killeen, N. 1996, Multichannel Image Reconstruction, Image Analysis and Display (MIRIAD) Users Guide, ATNF, CSIRO

Slee, O. B., \& Stewart, R. T. 1989, MNRAS, 236, 129

Slee, O. B., Nelson, G. J., Innis, J. L., Stewart, R. T., Vaughan, A. E., \& Wright, A. E. 1986, PASA, 6, 312

Tagliaferri, G., Covino, S., Fleming, T. A., Gagné, M., Pallavicini, R., Haardt, F., \& Uchida, Y. 1997, A\&A, 321, 850

Watson, L. 1999, PhD Thesis, University of Canterbury

Zuckerman, B., Song, I., Bessell, M., \& Webb, R. 2001, ApJ, 562, L87 